\section{Sporadic sexual reproduction Sex to some degree}

\section{LW Beukeboom} January 2007

$\mathrm{T}$ he evolution of sex has been considered the queen of problems in evolutionary biology (Bell, 1982). The problem is why the male sex has not been discarded as it does not serve any short-term benefits. Males do not produce offspring and the energy saved on producing males could be allocated to females that can reproduce by themselves. On the other hand, sex may be beneficial in the long term because it creates new variation for selection to act upon (Weismann, 1889). A longstanding question is why there are so few organisms that solve this paradox with a little bit of sex (Green and Noakes, 1995), by combining the benefits of occasional sexual reproduction with low investment in male function? A recent study (D'Souza et al., 2006) shows that a freshwater flatworm may thrive from predominantly parthenogenetic reproduction mixed with a little bit of sex.

Flatworms of the species Schmidtea polychroa do it quite differently. First, they are simultaneous hermaphrodites, meaning that every individual worm is both male and female during its entire lifespan. Second, they require sperm entering the egg to initiate egg devel- opment, but the sperm chromosomes are typically expelled from the zygote and do not contribute genetically to the next generation. Hence, reproduction is parthenogenetic and sperm dependent. Third, parthenogens are polyploid, usually triploid, but sometimes tetraploid. They produce polyploid eggs, but haploid sperm (Benazzi Lentati, 1970). Finally, although each individual has male and female reproductive organs, they cannot fertilize themselves, but need to obtain sperm from a conspecific individual. Why go through all this hassle to reproduce?

D'Souza et al. (2006) fingerprinted parental worms and their progeny to show that paternal DNA is sometimes inherited (Figure 1). They combined this with karyological analyses and showed that triploid mothers can produce tetraploid offspring with added paternal microsatellite alleles. Although such genome additions had been reported before, it is now clear that the added chromosome set need not be of maternal origin, for example, resulting from meiotic aberrations during oogenesis. Even more surprisingly, the authors also observed paternal inheritance with-

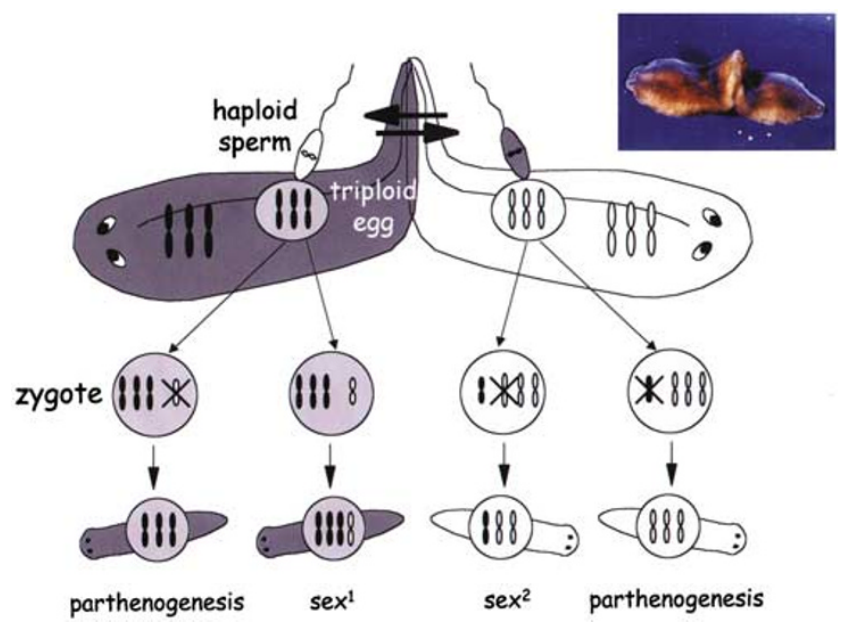

Figure 1 Reproduction in S. polychroa. Hermaphrodites are polyploid and exchange sperm reciprocally. Sperm is either used for egg activation only and reproduction is clonal (spermdependent parthenogenesis, outer diagrams) or, occasionally, contributes genetically to offspring and reproduction is sexual (inner diagrams). Paternal inheritance can increase ploidy (type 1) or be accompanied by maternal chromosome displacement (type 2). Worm drawing and photo - Nico Michiels.

out an increase in offspring ploidy. In several crosses they found that addition of paternal alleles was compensated by loss of maternal alleles. Overall, the authors estimate that $5 \%$ of the offspring is not clonally produced. These observations alter our view of spermdependent parthenogenesis, it may be considered as a means of 'minimal sex'.

Although not a common mode of reproduction, sperm-dependent parthenogenesis has evolved multiple times within seven phyla (Beukeboom and Vrijenhoek, 1998). It may simply be considered as an evolutionary transition towards obligate parthenogenesis, but it has also been questioned whether such a reproduction mode would confer any selective advantage. For S. polychroa this question can now be answered positively; it allows for occasional sexual recombination that generates genetic variation. It remains to be seen whether this is a common phenomenon among sperm parthenogens in different taxa.

A number of intriguing follow-up questions remain. From a mechanistic point of view, how do these worms control paternal inheritance? Are the observed cases merely accidental byproducts of an imperfect sperm expulsion mechanism or can the incorporation of paternal chromosomes be actively manipulated? Is paternal inheritance always involving complete chromosome sets, or is the process more intricate and allowing for replacement of single chromosomes or even recombination between egg and sperm chromosomes? Answering these questions requires more genetic experiments and detailed marker screening of parents and offspring. A first approach towards determining the potential adaptive significance of the process is to look for genetic variation for rare sex. Frequencies of paternal inheritance varied from 0 to $25 \%$ per investigated family. This suggests that selection for increased paternal inheritance may be effective. More challenging would be to show that populations that undergo paternal inheritance are selectively favoured over pure clonal ones. They may be better in preventing mutation accumulation or in resisting strong environmental perturbations, as predicted by general theories about the evolution of sex.

It has been a longstanding enigma why so few organisms exist that combine parthenogenetic and sexual reproduction. A number of recent studies have shown that parthenogens can have covert sex and suggest that rare sexual processes may be more common than previously thought. The flatworm 
S. polychroa is a good example of an organism with, at first sight, an unnecessarily complicated reproductive mode, but at deeper insight it may well be efficiently exploiting the benefits of sexual and asexual reproduction.

LW Beukeboom is at the Centre for Ecological and Evolutionary Studies, University of Groningen, PO Box 14, NL-9750 AA Haren, The Netherlands.

e-mail: l.w.beukeboom@rug.nl

Bell G (1982). The Masterpiece of Nature: The Evolution and Genetics of Sexuality. University of California Press: Berkeley.
Benazzi Lentati G (1970). Gamatogenesis and egg fertilization in planarians. Int Rev Cytol 27: 101-179.

Beukeboom LW, Vrijenhoek RC (1998). Evolutionary genetics and ecology of spermdependent parthenogenesis. J Evol Biol 11 755-782.

Green RF, Noakes DLG (1995). Is a little bit of sex as good as a lot? Theor Biol 174 87-96.

D'Souza TG, Schulte RD, Schulenberg H, Michiels NK (2006). Paternal inheritance in parthenogenetic forms of the planarian Schmidtea polychroa. Heredity 97: 97-101.

Weismann A (1889). Essays upon Heredity and Kindred Biological Problems. Clarendon Press: Oxford.

\section{Editor's suggested reading}

Maynard-Smith JM (1978). The Evolution of Sex. Cambridge University Press: Cambridge.

Medrano M, Alonso C, Herrera CM (2005). Mating system, sex ratio, and persistence of females in the gynodioecious shrub Daphne laureola L. (Thymelaeaceae). Heredity 94: 37-43.

Pomiankowski A, Bridle J (2004). No sex please we're at QLE (quasi-linkage equilibrium). Heredity 93: 407.

Suomalainen E, Saura A, Lokki J (1987). Cytology and Evolution in Parthenogenesis. CRC Boca Raton: Florida.

West SA, Lively CM, Read AF (1999). A pluralistic approach to sex and recombination. J Evol Biol 12: $1003-1012$ 